\title{
CONTINUOUS IMAGES OF ORDERED COMPACTA AND A NEW DIMENSION WHICH NEGLECTS METRIC SUBCONTINUA
}

\author{
BY \\ SIBE MARDEŠIĆ \\ Dedicated to Professor A. D. Wallace for his 60 th birthday
}

All spaces in this paper are assumed to be topological Hausdorff spaces. An ordered compactum is a compact space $K$ (possibly nonmetrizable) provided with a total ordering $<$ such that the topology of $K$ is the topology induced by the ordering <. If the ordered compactum is in addition connected, we have an ordered continuum $C$. The class of all spaces $X$ which can be obtained as the image $f(K)$ of an ordered compactum $K$ under a continuous mapping $f: K \rightarrow X$ onto $X$ (and similarly the class of images of ordered continua $C$ ) has been studied in the last few years in particular by P. Papic' and the author (cf. [2]-[8]) and by L. B. Treybig (cf. [9], [10]). Although several interesting properties of these spaces were found (all indicating that the class of these spaces may be smaller than one might have suspected), the characterization of these spaces remains still open. One of the difficulties appears to be a lack of proper examples, in particular in dimensions higher than 1.

In known examples, dimension higher than 1 is always due to the presence of higher dimensional metric subcontinua, thus suggesting that images of ordered compacta are essentially of dimension $\leqq 1$, provided metric subcontinua be disregarded. The main purpose of this paper is to give a precise meaning to this statement and then to prove it.

1. A reduced dimension which neglects metric subcontinua. Statement of the main theorem. In this section we define, for Hausdoff compact spaces only, a notion of reduced dimension which neglects metric subcontinua. It assigns to every compactum $X$ an integer Ind $(X, \mathfrak{M}) \geqq-1$, where $\mathfrak{M}$ denotes the class of metric continua. As usually the definition is by induction.

Definition 1. Let $X$ be a Hausdorff compact space. $\operatorname{Ind}(X, \mathfrak{M})=-1$ means that $X$ is empty. We say that $\operatorname{Ind}(X, \mathfrak{M}) \leqq 0$ provided all components of $X$ are metric continua. We say that Ind $(X, \mathfrak{M}) \leqq n, n>0$, provided for every nonempty closed subset $F \subset X$ and every open set $U$ of $X$ containing $F$ there exists an open set $V$ of $X, F \subset V \subset U$, such that $\operatorname{Ind}(\operatorname{Fr} V, \mathfrak{M}) \leqq n-1$. If Ind $(X, \mathfrak{M}) \leqq n$, but Ind $(X, \mathfrak{M}) \leqq n-1$ does not hold, then we say that $\operatorname{Ind}(X, \mathfrak{M})=n$.

Presented to the Society, April 12, 1965 ; received by the editors May 19, 1965. 
The value Ind $(X, \mathfrak{M})$ is clearly well defined. Comparing $\operatorname{Ind}(X, \mathfrak{M})$ with the usual definition for large inductive dimension Ind $X$, we immediately find that

(1) $\operatorname{Ind}(X, \mathfrak{M} \imath) \leqq \operatorname{Ind} X$.

Any metric continuum $X$ of dimension Ind $X=\operatorname{dim} X>1$ is an example of a compactum where Ind $(X, \mathfrak{M})<$ Ind $X$, because Ind $(X, \mathfrak{M})=0$. For a nonmetrizable continuuum $X$ we have Ind $(X, \mathfrak{M}) \geqq 1$. If a compactum $X$ contains no metrizable nondegenerate subcontinuum, then

(2) $\operatorname{Ind}(X, \mathfrak{M})=$ Ind $X$.

Ind $(X, \mathfrak{M})$ is a monotonously increasing function on the set of closed subsets of $X$ (ordered by inclusion $\subset$ ). Ind $(X, \mathfrak{M})$ does not satisfy the sum theorem. For example, let $\Omega=\left\{\alpha \mid \alpha<\omega_{1}\right\}$ be the set of all countable ordinals and let $X$ be the ordered continuum obtained by ordering lexicographically the product

$$
\Omega \times[0,1)
$$

and adjoining a last point $\omega_{1}$. Clearly $X=X_{1} \cup X_{2}$, where $X_{1}$ is the closure of the union of all segments of $X$ of the form $\left[\alpha \times 0, \alpha \times \frac{1}{2}\right]_{X}, \alpha<\omega_{1}$, and $X_{2}$ is the closure of the union of all segments of $X$ of the form $\left[\alpha \times \frac{1}{2},(\alpha+1) \times 0\right]_{X}$, $\alpha<\omega_{1}$. Both $X_{1}$ and $X_{2}$ consist of $\aleph_{1}$ components which are real line segments or single points. Hence, Ind $\left(X_{i}, \mathfrak{M}\right)=0, i=1,2$. Nevertheless, Ind $(X, \mathfrak{M})=1$.

REMARK 1. Clearly, we could in the same way define a reduced small inductive dimension Ind $(X, \mathfrak{M}) \leqq \operatorname{Ind}(X, \mathfrak{M})$. Furthermore, instead of disregarding metric subcontinua, we could disregard subcontinua whose weight does not exceed a given cardinal $k$ and thus obtain a reduced dimension Ind $(X, k)$. Our previous definition would reduce to $\operatorname{Ind}\left(X, \aleph_{0}\right)$.

Now the main result can be stated as

THEOREM 1. If $X$ is a continuous image of an ordered compactum $K$, then its reduced dimension (modulo metric subcontinua) Ind $(X, \mathfrak{M}) \leqq 1$.

COROLlaRY 1. Let $X$ be a continuous image of an ordered compactum. If all metric subcontinua of $X$ are degenerate, then $\operatorname{dim} X=\operatorname{ind} X=\operatorname{Ind} X \leqq 1$.

REMARK 2. For each $n$ there exist examples of spaces $X$ with Ind $X=n$ such that $X$ is an image of an ordered continuum and contains no open metric subset.

Indeed, let $C$ be an ordered continuum which is nonmetric and has the property that any nondegenerate subcontinuum of $C$ is homeomorphic with $C$ itself. Such ordered continua do exist (cf., e.g., [1]). Clearly, $C$ has no metric open subset. Let $P$ be a metric Peano continuum of dimension $n$ and let $D$ be a countable subset $\left\{d_{1}, d_{2}, \cdots\right\}$ of $P$ dense on $P$. Consider a sequence $C_{i}, i=1,2, \cdots$, of copies of $C$ and denote by $c_{i}$ one of the two endpoints of $C_{i}$. Let $X$ be the subset of the Cartesian product $P \times \prod_{i=1}^{\infty} C_{i}$ given by 
(3) $\left.X=\left[P \times c_{1} \times c_{2} \times \cdots\right] \cup \bigcup_{i=1}^{\infty}\left(d_{i} \times c_{1} \times \cdots \times c_{i-1} \times C_{i} \times c_{i+1} \times \cdots\right)\right]$.

Clearly, $X$ is a continuum and has all the required properties.

REMARK 3. Let $Y$ be a compact space with the property that all metric subcontinua of $Y$ are of dimension $\leqq n$ and Ind $(Y, \mathfrak{M}) \leqq n$. One cannot conclude that Ind $Y \leqq n$. A counterexample, for $n=1$, is the chainable continuum described in $\S 4$ of [2], whose large inductive dimension Ind is greater than 1.

QUESTION 1. Let $X$ be an image of an ordered compactum and let every metric subcontinuum $M \subset X$ be of dimension $\operatorname{dim} M \leqq n, n \geqq 1$. Does it follow that Ind $X \leqq n ?$

2. Open $F_{\sigma}$-sets and their frontiers. Let $s(X)$ denote the density (or degree of separability) of $X$, i.e. the minimal number of points in a set dense in $X$. In order to Theorem 1 we first prove

THEOREM 2. Let $X$ be a continuous image of an ordered compactum $K$ and let $U \subset X$ be an open set in $X$. If $U$ is the union of $\leqq \kappa$ closed subsets of $X$ ( $\kappa$ an infinite cardinal), then the density of its frontier

$$
s(\operatorname{Fr} U) \leqq \kappa .
$$

Proof of Theorem 2. Let $X=f(K)$ and let

(1) $U=\bigcup_{\alpha} F_{\alpha}, \alpha \in A, F_{\alpha}=\mathrm{Cl} F_{\alpha} \subset X$,

where the cardinal $K(A) \leqq \kappa$. The open set $f^{-1}(U)$ decomposes into nonempty disjoint maximal intervals

(2) $U_{j}=\left(a_{j}, b_{j}\right)_{K}=\left\{t \mid t \in K, a_{j}<t<b_{j}\right\}, j \in J$,

where $a_{j}\left(b_{j}\right)$ is the empty symbol if $U_{j}$ is an interval containing the first (last) endpoint of $K$. Due to compactness, $f^{-1}\left(F_{\alpha}\right), \alpha \in A$, intersects only a finite number of intervals $U_{j}$ and each $U_{j}$ is intersected by at least one set $f^{-1}\left(F_{\alpha}\right), \alpha \in A$. Consequently, $k(J) \leqq \kappa$. Therefore, the set $D \subset K$ of all the endpoints $a_{j}, b_{j}$ of $U_{j}, j \in J$, is of power

(3) $k(D) \leqq \kappa$.

In order to prove Theorem 2 it suffices to prove that

(4) $\operatorname{Fr} U \subset f(\mathrm{Cl} D) \subset \mathrm{Cl} f(D)$.

Indeed, we know that the degree of separability $s$ is a monotonously increasing function on closed subsets of images of ordered compacta (see Theorem 12 in [7]). Therefore, (4) implies

(5) $s(\mathrm{Fr} U) \leqq s(\mathrm{Cl} f(D)) \leqq k(f(D)) \leqq k(D) \leqq \kappa$.

In order to prove (4) first observe that

(6) $\operatorname{Fr} f^{-1}(U) \subset \mathrm{Cl} D$.

Indeed, let $t_{0} \in \operatorname{Fr} f^{-1}(U)$. If $t_{0}$ is not an endpoint of $K$, then for every interval $\left(k_{0}, k_{1}\right)_{K}, k_{0}<k_{1}, k_{0}, k_{1} \in K$, containing $t_{0}, k_{0}<t_{0}<k_{1}$, there is a $j \in J$ such that $\left(k_{0}, k_{1}\right)_{K} \cap U_{j} \neq 0$, because $t_{0} \in \mathrm{Cl} f^{-1}(U)=\mathrm{Cl}\left(\bigcup_{j}^{\prime} U_{j}\right)$. However, $\left(k_{0}, k_{1}\right)_{K}$ cannot be contained in $U_{j}$, because $t_{0} \notin f^{-1}(U)$. Consequently, if $a_{j} \leqq k_{0}$, then 
$k_{0}<b_{j}<k_{1}$, and if $k_{0}<a_{j}$, then $a_{j}<k_{1}$. Thus, $\left(k_{0}, k_{1}\right)_{K} \cap D \neq 0$, for $a_{j}, b_{j} \in D$. In the case when $t_{0}$ is the first (last) endpoint of $K$, a similar argument proves that $\left(, k_{1}\right)_{K} \cap D \neq 0\left(\left(k_{0},\right)_{K} \cap D \neq 0\right)$, for any $k_{1} \in K, t_{0}<k_{1}\left(k_{0} \in K, k_{0}<t_{0}\right)$. Thus, (6) is established.

(6) reduces the proof of (4) to proving

(7) $\operatorname{Fr} U=f\left(\operatorname{Fr} f^{-1}(U)\right)$,

which is an easy consequence of the fact that $f\left(\mathrm{Cl}^{-1}(U)\right)$ is a closed set which contains $U$ and therefore coincides with $\mathrm{Cl} U$.

COROllary 2. Every open $F_{\sigma}$-set $U \subset X=f(K)$ has a separable frontier, i.e. $s(\operatorname{Fr} U) \leqq \kappa_{0}$.

Remark 4. Examples show that Fr $U$ of an open $F_{\sigma}$-set in the image $X$ of an ordered compactum may not be metric. Indeed, let $X$ be the ordered compactum obtained by replacing each rational point $t$ of $I=[0,1]$ by a copy $I_{t}$ of $I$ and by replacing each irrational point $t$ of $I$ by two points $\{0,1\}$. Let $U$ be the union of all the interiors Int $I_{t}$ of the intervals $I_{t}$, when $t$ runs through the set of all rationals. Each Int $I_{t}$ being an open $F_{\sigma}$-set, $U$ is itself an open $F_{\sigma}$-set in $X$. However, the frontier of $U$ is not metrizable.

QUESTION 2. Let $X$ be the image of an ordered compactum and let $U$ be a connected open $F_{\sigma}$-set in $X$. Is Fr $U$ metrizable?

3. Proof of Theorem 1. Let $X$ be the image of an ordered compactum $K$ under a continuous mapping $f: K \rightarrow X$, let $F \subset X$ be a closed subset and $U \subset X$ an open subset, $F \subset U$. Using normality we can construct, by induction, a sequence of open sets $V_{n}, n=1,2, \cdots$, such that

(1) $F \subset V_{1} \subset \mathrm{Cl} V_{1} \subset \cdots \subset V_{n} \subset \mathrm{Cl} V_{n} \subset V_{n+1} \subset \cdots \subset U$.

Let

(2) $V=\bigcup_{n=1}^{\infty} V_{n}$.

Clearly,

(3) $F \subset V \subset U$.

Moreover, $V$ is an $F_{\sigma}$-set because

(4) $V=\bigcup_{n=1}^{\infty} \mathrm{Cl} V_{n}$.

Consequently, by Corollary 2, Fr $V$ is separable. The density $s$ being monotone on closed subsets of $X$ (Theorem 12 in [7]), we conclude that each component $X_{\alpha}$ of FrV is separable too. However, $X_{\alpha}$ is itself an image of the ordered compactum $K_{\alpha}=f^{-1}\left(X_{\alpha}\right)$ and is in addition connected and separable. Therefore, by a theorem of L. B. Treybig (Theorem 1 of [10]), $X_{\alpha}$ must be metric, which proves that $\operatorname{Ind}(\operatorname{Fr} V, \mathfrak{M})=0$, i. e. that $\operatorname{Ind}(X, \mathfrak{M}) \leqq 1$.

The precise statement of Treybig's theorem is the following:

Theorem 3 (L. B. Treybig). Let $X$ be the image of an ordered compactum, let 
$w(X)$ denote the weight $\left.{ }^{1}\right)$ of $X$ and $s(X)$ the density. If $X$ is connected, then $s(X)=w(X)$.

The theorem was proved by P. Papić and the author first for images of ordered continua [5] and then for locally connected images of ordered compacta [7]. The general case was obtained by Treybig in [10].

In the next two sections 4 and 5, we give a new proof of this basic result, which we believe is considerably simpler than Treybig's original proof. In particular in \$4. We associate with every map $f: K \rightarrow X$ onto $X$ a family $₹$ of closed sets $F \subset X$ which plays an important role in proving results of this type.

REMARK 5. The proof of Theorem 1 given in the present section also demonstrates that any two closed disjoint subsets $F_{1}, F_{2} \subset X$ can be separated by a separable compact subset $M$ of $X$. This suggests the following

Question 3. Is it always possible to separate $F_{1}$ from $F_{2}$ by a metric compact subset $M$ of $X$ ?

4. The family $\mathfrak{F}$ of closed subsets of $X$ associated with a map $f: K \rightarrow X$. Given any (infinite) ordered compactum $K$ and a $\operatorname{map} f: K \rightarrow X$ onto a space $X$, we associate with $f$ a family $\mathfrak{F}$ (not uniquely determined) of closed subsets $F \subset X$ as follows. We choose a dense subset $D \subset K$ such that

(1) $k(D)=s(K)$.

F consists of all closed subsets $F \subset X$ of the form

(2) $F=f\left(\bigcup_{j}\left[c_{j}, d_{j}\right]_{K}\right), c_{j}, d_{j} \in D$, where $j$ runs through a finite set. Here $\left[c_{j}, d_{j}\right]_{K}$ denotes the segment $\left\{t \mid t \in K, c_{j} \leqq t \leqq d_{j}\right\}$; we also admit the case when $c_{j}$ or $d_{j}$ are emply symbols so that e.g. we have $\left[t, d_{j}\right]_{K}=\left\{t \mid t \in K, t \leqq d_{j}\right\}$. Clearly

(3) $k(\mathfrak{Y}) \leqq k(D)=s(K)$.

We shall prove two useful lemmas concerning the family $\mathfrak{F}\left({ }^{2}\right)$.

Lemma 1. Let $M$ be closed and $U$ an open subset of $X=f(K), M \subset U$. If there is no pair of consecutive points $a, b \in K, a<b,(a, b)_{K}=0$, such that $f$ maps one of them into $M$ and the other into $X\lceil U$, then there is a closed set $F \in \mathfrak{F}$ such that $M \subset F \subset U$.

Proof. Consider the closed set $f^{-1}(M)$ and the open set $f^{-1}(U)$ which contains it. The set $f^{-1}(U)$ is the union of maximal disjoint intervals $U_{j}=\left(a_{j}, b_{j}\right)_{K}, j \in J$; here $a_{j}$ and $b_{j}$ can be empty symbols in which case we obtain initial and terminal intervals. The set $f^{-1}(M)$ being compact, it meets only finitely many intervals $U_{j}, j=j_{1}, \cdots, j_{n}$. For each $j \in\left\{j_{1}, \cdots, j_{n}\right\}$, there is a minimal element $a_{j}^{\prime}$ of $U_{j} \cap f^{-1}(M)$, and a maximal element $b_{j}^{\prime}$ of $U_{j} \cap f^{-1}(M)$. By assumption, the interval $\left(a_{j}, a_{j}^{\prime}\right)_{K}$ cannot be empty, because $f\left(a_{j}^{\prime}\right) \in M$, while $f\left(a_{j}\right) \in X \backslash U$, for $a_{j} \notin f^{-1}(U)$. Hence, there is a point $c_{j} \in D$ such that $c_{j} \in\left(a_{j}, a_{j}^{\prime}\right)_{K}$. Similarly, we find a point

(1) The weight $w(X)$ is the least cardinal of a basis for the topology of $X$.

(2) The family $\mathfrak{F}$ was in fact introduced already in [5] (cf. the proof of Lemma 3). 
$d_{j} \in D$ súch that $d_{j} \in\left(b_{j}^{\prime}, b_{j}\right)_{K}$. However, if, e.g., $b_{j}$ is the empty symbol, we define only $c_{j}$ and take for $d_{j}$ the empty symbol. The corresponding set

$$
F=f\left(\bigcup_{k=1}^{n}\left[c_{j_{k}}, d_{j_{k}}\right]_{K}\right) \in \mathfrak{F}
$$

clearly has the required properties, because

$$
f^{-1}(M) \subset \bigcup_{k=1}^{n}\left[c_{j_{k}}, d_{j_{k}}\right]_{K} \subset f^{-1}(U) .
$$

REMARK 6. A statement stronger than Lemma 1 is proved by L. B. Treybig as Lemma 3 in [10].

Lemma 2. Let $M$ and $N$ be disjoint closed subsets of $X=f(K)$. Then there is a set $F \in \mathfrak{F}$ which separates $X$ between $M$ and $N$, i.e. $X \backslash F$ can be decomposed into two disjoint open sets $U, V$ such that $M \subset U, N \subset V$.

Proof. Choose open sets $W_{1}, W, W_{2}$ in $X$ in such a way that

(4) $M \subset W_{1} \subset \mathrm{Cl} W_{1} \subset W \subset \mathrm{Cl} W \subset W_{2} \subset \mathrm{Cl} W_{2} \subset X \backslash N$.

Consider the pair of closed disjoint sets

(5) $\left(\mathrm{Cl} W_{2}\right) \backslash W_{1}, M \cup N$.

Continuity of $f: K \rightarrow X$ implies that there are only finitely many pairs of consecutive points $a_{i}, b_{i} \in K, a_{i}<b_{i},\left(a_{i}, b_{i}\right)_{K}=0, i=1, \cdots, n$, such that $f$ maps one of the points into one of the sets (5) and the other point into the other set of (5) (cf. Lemma 4 of [7]). Some of the points $a_{i}, b_{i}, i=1, \cdots, n$, may have their $f$-images lying on the frontier Fr $W$; denote the images of these points by $x_{j}, j=1, \cdots, p$, $p \leqq 2 n$. Surround each $x_{j} \in \operatorname{Fr} W, j=1, \cdots, p$, by an open neighborhood $V_{j}$ such that $\mathrm{Cl} V_{j}$ lies in $W_{2} \mid \mathrm{Cl} W_{1}$, that all $\mathrm{Cl} V_{j}$ are disjoint and that $\mathrm{Cl} V_{j}$ contains only one of the points $f\left(a_{i}\right), f\left(b_{i}\right), i=1, \cdots, n$, namely $x_{j}$. Define a new open set $V$ by

(6) $V=W \cup V_{1} \cup \cdots \cup V_{p}$.

Clearly,

(7) $\operatorname{Fr} V \subset\left(\mathrm{Cl} W_{2}\right) \backslash W_{1} \subset X \backslash(M \cup N)$

and separates $X$ between $M$ and $N$, because $M \subset V$ and $N \subset X \backslash \mathrm{Cl} V$. Moreover, there is no pair of consecutive points $a, b \in K, a<b,(a, b)_{K}=0$, such that $f$ maps one of them into $M \cup N$ and the other one into $\operatorname{Fr} V \subset\left(\mathrm{Cl} W_{2}\right) \backslash W_{1}$. Therefore, by Lemma 1 , there is a set $F \in \mathfrak{F}$ such that

(8) Fr $V \subset F$

and

(9) $F \cap(M \cup N)=0$.

Clearly, $F$ separates $X$ between $M$ and $N$.

5. Proof of Theorem 3. The essential step consists in establishing

THEOREM $3^{\prime}$. Let $K$ be an ordered compactum and $f: K \rightarrow X$ a mapping onto $X$. If $X$ is :connected, isthen $w(X)] \leqq\left[s(K)\left({ }^{3}\right)\right.$.

(3) Compare with Lemma 3 of [5]. 
Proof of Theorem $3^{\prime}$. Let $\mathfrak{F}$ be the family of closed sets $F$ of $X$ associated with $f: K \rightarrow X$ as described in $\$ 4$. Clearly,

(1) $k(\mathfrak{F} \times \mathfrak{F})=k(\mathfrak{F}) \leqq s(K)$.

With each pair $\left(F_{1}, F_{2}\right) \in \mathfrak{F} \times \mathfrak{F}$ we associate a new pair of sets $F_{1}^{*}, F_{2}^{*} \subset X$ as follows. $F_{i}^{*}, i=1,2$, is the union of $F_{i}$ and of all the components $M_{\alpha}$ of $X /\left(F_{1} \cup F_{2}\right)$ which have the property that

(2) $F_{i} \cap \mathrm{Cl} M_{\alpha} \neq 0$.

$X$ being connected and compact, we have

(3) $X=F_{1}^{*} \cup F_{2}^{*}$.

Let $\mathfrak{B}$ be the family of all sets of the type Int $F_{i}^{*}, i=1,2,\left(F_{1}, F_{2}\right) \in \mathfrak{F} \times \mathfrak{F}$. Clearly, $\mathfrak{B}$ is a family of open sets and

(4) $k(\mathfrak{B}) \leqq k(\mathfrak{F} \times \mathfrak{F}) \leqq s(K)$.

The proof will be completed if we show that $\mathfrak{B}$ is a basis for the topology of $X$.

Let $x \in X$ and let $U$ be an open set of $X, x \in U \subset X$. Choose open sets $U_{1}, U_{2} \subset X$ such that

(5) $x \in U_{1} \subset \mathrm{Cl} U_{1} \subset U_{2} \subset \mathrm{Cl} U_{2} \subset U$.

By Lemma 2, we can choose closed sets $F_{1}, F_{2} \in F$ such that $F_{1}$ separates the pair $\mathrm{Cl} U_{1}, X \backslash U_{2}$, while $F_{2}$ separates the pair $\mathrm{Cl} U_{2}, X \backslash U$. If a component $M_{\alpha}$ of $X \backslash\left(F_{1} \cup F_{2}\right)$ intersects $\mathrm{Cl} U_{1}$, then it cannot meet $X \backslash U_{2}$ and, therefore, $M_{\alpha} \subset U_{2}$. Consequently, $\mathrm{Cl} M_{\alpha} \subset \mathrm{Cl} U_{2}$ and thus $F_{2} \cap \mathrm{Cl} M_{\alpha}=0$. This means, by (3), that $F_{1} \cap \mathrm{Cl} M_{\alpha} \neq 0$ and therefore $M_{\alpha} \subset F_{1}^{*}$. Since $\mathrm{Cl} U_{1} \subset X \mid\left(F_{1} \cup F_{2}\right)$, we obtain

(6) $U_{1} \subset \mathrm{Cl} U_{1} \subset F_{1}^{*}$.

On the other hand, if $M_{\alpha} \subset F_{1}^{*}$, i.e. $F_{1} \cap \mathrm{Cl} M_{\alpha} \neq 0$, then a fortiori

(7) $U_{2} \cap \mathrm{Cl} M_{\alpha} \neq 0$,

because $F_{1} \subset U_{2}$. However, (7) implies

(8) $U_{2} \cap M_{\alpha} \neq 0$

and a fortiori

(9) $M_{\alpha} \cap \mathrm{Cl} U_{2} \neq 0$.

Hence, $M_{\alpha}$ cannot meet $X \backslash U$, which proves that

(10) $M_{\alpha} \subset U$.

Since also $F_{1} \subset U$, it follows that

(11) $F_{1}^{*} \subset U$.

Now (6) and (11) imply

(12) $x \in U_{1} \subset \operatorname{Int} F_{1}^{*} \subset U$,

proving thus that $\mathfrak{B}$ is indeed a basis.

Proof of Theorem 3. It suffices to observe that $X$ can always be obtained as the image of an ordered compactum $K$ for which $s(K)=s(X)$ (see Corollary 5 in [7]). Then, Theorem $3^{\prime}$ proves that $w(X) \leqq s(K)=s(X)$, which completes the proof, because $s(X) \leqq w(X)$ always holds good. An alternative consists in applying Lemma 4 of [9], which enables one to assume that $f: K \rightarrow X$ is irreducible, i.e., 
$f$ maps no closed proper subset of $K$ onto $X$. Then again $s(K)=s(X)$ (Lemma 1 in [10]).

These remarks combined with Lemma 2 also establish

THEOREM 4. Let $X$ be an image of an ordered compactum. Then there exists a family $\mathfrak{F}$ of closed subsets $F \subset X$ such that its power $k(\mathfrak{F}) \leqq s(X)$ and that $X$ can be separated between any two disjoint closed subsets $M, N \subset X$ by some member $F$ of $\mathfrak{F}$.

6. Products $X \times Y$ as images of ordered compacta. The following theorem has been proved independently by A. J. Ward (unpublished) and L.B. Treybig [9]( $\left.{ }^{4}\right)$.

Theorem 5 (A. J. WARd, L. B. Treybig). Let $X$ and $Y$ be infinite compacta. If $X \times Y$ is the image of an ordered compactum $K$, then both $X$ and $Y$ are metric.

Corollary 2 and Lemma 1 of this paper provide means for a new and very simple proof of Theorem 5 .

Proof of Theorem 5. Using the fact that every ordered compactum $K$ is sequentially compact, we conclude that $Y$ is itself sequentially compact, because it is obviously an image of $K$. Consequently, there is no loss of generality in assuming that $Y$ is a monotonously increasing sequence of different real numbers $y_{1}<y_{2}<\cdots<y_{n}<\cdots$ tending towards $y_{\infty}$ (cf. [9]).

Clearly, the sets

(1) $U_{n}=X \times\left\{y_{1}, \cdots, y_{n}\right\}$

form a sequence of closed and open sets whose union is the open set

(2) $U=X \times\left(Y \backslash\left\{y_{\infty}\right\}\right)$.

$U$ is therefore an open $F_{\sigma}$-set whose frontier $X \times\left\{y_{\infty}\right\}$ must be separable, by Corollary 2. This proves that $X$ itself, and therefore also $X \times Y$, are separable. Thus there is no loss of generality in assuming that $K$ itself is separable (cf. the end of $\S 5$ ).

Now consider the family $\mathfrak{F}$ of closed sets of $X \times Y$ associated with $f: K \rightarrow X \times Y$ following $\$ 4$. Observe that

(3) $k(\mathfrak{F}) \leqq \aleph_{0}$

Let $\pi: X \times Y \rightarrow X$ denote the natural projection and let $\mathfrak{B}$ be the family of all sets of the form

(4) Int $\pi(F), \quad F \in \mathfrak{F}$.

Clearly, $\mathfrak{B}$ is a countable family of open sets of $X$ and it suffices to prove that $\mathfrak{B}$ is a basis for $X$.

Indeed, let $x \in X$ and let $V$ be an open set in $X, x \in V \subset X$. Take another open set $W \subset X$ such that

(5) $x \in W \subset \mathrm{Cl} W \subset V$.

(4) The case when $X \times Y$ is the image of an ordered continuum has been proved earlier in [5]. 
Consider closed disjoint sets $\pi^{-1}(\mathrm{Cl} W)$ and $\pi^{-1}(X \backslash V)$. Since $f$ is continuous, there exist at most finitely many pairs of consecutive points $a_{i}, b_{i} \in K, a_{i}<b_{i}$, $\left(a_{i}, b_{i}\right)_{K}=0$, such that $f$ maps one point into $\pi^{-1}(\mathrm{Cl} W)$ and the other point into $\pi^{-1}(X \backslash V)$. Therefore, we can choose an index $n<\infty$ such that $(\mathrm{Cl} W) \times\left\{y_{n}\right\}$ is free of images of these points. This enables us to apply Lemma 1 of $\S 4$ and find a set $F \in \mathfrak{F}$ such that

(6) $(\mathrm{Cl} W) \times\left\{y_{n}\right\} \subset F \subset \pi^{-1}(V)$.

Applying $\pi$ to (6) we obtain

(7) $W \subset \mathrm{Cl} W \subset \pi F \subset V$,

which proves that

(8) $x \in \operatorname{Int} \pi F \subset V$,

thus demonstrating that $\mathfrak{B}$ is indeed a basis for $X$.

7. An alternative proof for Theorem 5 . We conclude this paper by giving yet another proof of Theorem 5 which has the advantage of being straightforward and short. We assume again that $Y$ is of the form $\left\{y_{1}, \cdots, y_{n}, \cdots, y_{\infty}\right\}$.

Consider, for each $n<\infty$, the set $X \times\left\{y_{n}\right\}$ which is closed and open in $X \times Y$. Consequently, the set

(1) $K_{n}=f^{-1}\left(X \times\left\{y_{n}\right\}\right)$

is also closed and open, and therefore a union of finitely many intervals $I^{n}, \cdots, I_{k_{n}}^{n}$ each of which is closed and open. Notice that for different $n, n^{\prime}$ the intervals $I_{m}^{n}$ and $I_{m^{\prime}}^{n^{\prime}}$ are disjoint. Let $\pi: X \times Y \rightarrow X$ denote the natural projection. For each $n<\infty$ consider subsets $\left\{m_{1}, \cdots, m_{s}\right\} \subset\left\{1, \cdots, k_{n}\right\}$ and with each of them associate the open set

(2) $U_{m_{1} \ldots m_{s}}^{n}=\operatorname{Int} \pi\left[f\left(I_{m_{1}}^{n}\right) \cup \ldots \cup f\left(I_{m_{s}}^{n}\right)\right]$.

Clearly, we obtain a countable collection $\mathfrak{B}$ of open sets of $X$. It suffices to prove that $\mathfrak{B}$ is a basis for $X$.

Let $x \in X$ and let $U \subset X$ be an open set about $x$. Choose an open set $V$ from $X$ such that $x \in V \subset \mathrm{Cl} V \subset U$ and consider, for each $n<\infty$, the set $(\mathrm{Cl} V) \times\left\{y_{n}\right\} \subset X \times\left\{y_{n}\right\}$. Choose the subset $M_{n}=\left\{m_{1}, \cdots, m_{s(n)}\right) \subset\left\{1, \cdots, k_{n}\right\}$ so as to consist precisely of those indexes $m$ for which

(3) $f\left(I_{m}^{n}\right) \cap\left[(\mathrm{Cl} V) \times\left\{y_{n}\right\}\right] \neq 0$.

Clearly,

(4) $\bigcup_{i=1}^{s(n)} f\left(I_{m_{i}}^{n}\right) \supset(\mathrm{Cl} V) \times\left\{y_{n}\right\}$, because

(5) $(\mathrm{Cl} V) \times\left\{y_{n}\right\} \subset X \times\left\{y_{n}\right\} \subset \bigcup_{m=1}^{k_{n}} f\left(I_{m}^{n}\right)$.

The intervals $I_{m}^{n}, m \in M_{n}, n=1,2, \cdots$, form a countable collection of disjoint intervals having the property that

(6) $f\left(I_{m}^{n}\right) \cap[(\mathrm{Cl} V) \times Y] \neq 0$.

Therefore, for all but finitely many, we must have

(7) $f\left(I_{m}^{n}\right) \subset U \times Y$

(cf. Lemma 4 of [7]). This proves that, for a sufficiently large $n$, we have 
(8) $\bigcup_{i=1}^{s(n)} f\left(I_{m_{i}}^{n}\right) \subset U \times Y$.

By (4), we conclude that

(9) $\pi\left[\bigcup_{i=1}^{s(n)} f\left(I_{m_{i}}^{n}\right)\right] \supset \mathrm{Cl} V$

and therefore

(10) $x \in V \subset U_{m_{1} \ldots m_{s(n)}}^{n}$.

On the other hand, (8) proves that

(11) $U_{m_{1} \ldots m_{s(n)}}^{n} \subset U$.

This completes the proof.

The author is indebted to Professor P. Papic of Zagreb University for many useful discussions concerning the subject as well as to Dr. G. T. Roberts of Hull University for a stimulating conversation leading to the concept of dimension neglecting metric subcontinua. The author wishes also to point out that by courtesy of Professor L. B. Treybig of Tulane University he had the privilege to read the manuscript of [10] before publication.

\section{REFERENCES}

1. R. Arens, On the construction of linear homogeneous continua, Bol. Soc. Mat. Mexicana 2 (1945), 33-36.

2. S. Mardešić, Chainable continua and inverse limits, Glasnik Mat.-Fiz. Astronom. 14 (1959), 219-232.

3. - On the Hahn-Mazurkiewicz theorem in nonmetric spaces, Proc. Amer. Math. Soc. 11 (1960), 929-937.

4. - Mapping ordered continua onto product spaces, Glasnik Mat.-Fiz. Astronom. 15 (1960), 85-89.

5. S. Mardešié and P. Papié, Continuous images of ordered continua, Glasnik Mat. - Fiz. Astronom. 15 (1960), 171-178.

6. —— Diadičeskie bikompakty i nepreryvnye otobraženija uporjadočennyh bikompaktov, Dokl. Akad. Nauk SSSR 143 (1962), 529-531.

7. - Continuous images of ordered compacta, the Suslin property and diadic compacta, Glasnik Mat.-Fiz. Astronom. 17 (1962), 3-25.

8. - Neki problemi preslikavanja uredenih kompakata (Some problems concerning mappings of ordered compacta), Matematićka Biblioteka (Beograd) 25 (1963), 11-22.

9. L. B. Treybig, Concerning continuous images of compact ordered spaces, Proc. Amer, Math. Soc. 15 (1964), 866-871.

10. - Concerning continua which are continuous images of compact ordered spaces, Duke Math. J. 32 (1965), 417-422.

\section{UNIVERSITY OF ZAGREB}

ZAGREB, YUgosLAVIA 